\title{
Las ciencias sociales en la encrucijada actual
}

\author{
Roberto Follari \\ Universidad Nacional de Cuyo, Mendoza, Argentina. \\ Email: rfollari@gmail.com
}

\begin{abstract}
Resumen: Las ciencias sociales son lentas para asumir temas relevantes: así seguridad, narcotráfico, cultura mediática, incluso neopopulismo y actuales gobiernos latinoamericanos, son temáticas donde el desarrollo teórico y empírico está muy por debajo de lo necesario. A la vez, posiciones como la de Wallerstein, han alertado acerca de los problemas epistemológicos que se derivan de tomar al Estado/Nación como unidad de análisis de lo social, lo cual -al reemplazar al siste$\mathrm{ma}$ /mundo como objeto- trae severos problemas, los cuales se han expresado también en las limitaciones para advertir el por qué de los fracasos del socialismo en sus realizaciones históricas concretas. La superación de esos problemas, aparece en Wallerstein menos clara que su diagnóstico: propuestas como la de interdisciplina resultan en ese caso poco fundadas y muy cercanas al sentido común, perdiéndose en ellas, incluso, el filo crítico-ideológico que subtiende a las posiciones de un autor que trabaja dentro del marxismo.
\end{abstract} interdisciplina.

Palabras clave: ciencias sociales, Wallerstein, Estado/Nación, socialismo,

\section{Social sciences in the current crossroads}

Abstract: Social sciences are slow to take up relevant issues: thus, security, drug trafficking, media culture, even neopopulism and current Latin American governments are topics where theoretical and empirical development is far below from what is needed. At the same time, positions, such asWallerstein's, have warned aboutepistemological problems that result from taking the State/Nation as a unit of social analysis. In doing so -when replacing the system/world as an object-appear severe problems that also expressthemselves in the limitations onhaving warnedaboutthe reasons for the failure of socialisms in their concrete historical realizations. The overcoming of these problems appearin Wallersteinless clear than his diagnosis: proposals such as interdisciplineappear in this case are poorly founded and very close to common sense, losing in them even the critical-ideological edge subtending the positions of an author who works within Marxism.

Keywords: social sciences, Wallerstein, State/Nation, socialism, interdisciplinary studies,

\section{As ciências sociais na encruzilhada atual}

Resumo: As ciências sociais são lentas para assumir as questões relevantes: segurança, tráfico de drogas, cultura de mídia, mesmo neopopulismo e atuais governos latino-americanos são temas onde o desenvolvimento teórico e empírico se encontra muito abaixo do que é necessário. Ao mesmo tempo, posições como de 
Wallerstein, têm alertado sobre os problemas epistemológicos que resultam de se levar o Estado/Nação como unidade de análise do social, que -ao substituir o siste$\mathrm{ma}$ /mundo como objeto- traz graves problemas, os quais também são expressos nas limitações para advertir as razões do fracasso do socialismo em suas realizações históricas concretas. A superação desses problemassurge menos clara em Wallerstein que no seu diagnóstico: propostas como a interdisciplinariedade resultam, neste caso, infundadas e muito próximas ao senso comum, perdendou-se nelas, inclusive o filão crítico-ideológico que subtende as posições de um autor que trabalha dentro do marxismo.

Palavras-chave: Ciências sociais, Wallerstein, Estado/Nação, socialismo, interdisciplinar.

Hace algunos años se lanzó el desafío. Diversos autores, algunos de ellos latinoamericanos (González Casanova, Boaventura de Sousa Santos) entendieron que estamos ante una nueva situación epocal. Que ella plantea nuevas exigencias conceptuales, y que las respuestas de las ciencias sociales a las mismas resultan anacrónicas, desajustadas y -en el mejor de los casos- incompletas.

La tarea de la Comisión Gulbenkian resultó importante al respecto, pues fue tomada como un hito. La asunción de que se estaba -ya desde los años noventa del siglo pasado- ante una situación inédita, y de que los supuestos epistémicos no habían tomado nota suficiente sobre la misma se volvió relativamente generalizada. Lo cual, por cierto, no significa que se hubiera transformado las prácticas intelectuales o institucionales, ni lo central de los marcos conceptuales que previamente las regían. Más bien se ha tratado de algo más vago; de un cierto desasosiego, de la imposibilidad de mantenerse satisfechos con las modalidades previas de trabajo sin que, por cierto, se encontrara otras suficientemente sólidas o -en algunos casossin que se asumiera el enorme esfuerzo de convertir a estas en un nuevo "habitus" intelectual.

Si bien sostenemos que no existen propiamente paradigmas en ciencias sociales (al menos en el sentido de Kuhn) ${ }^{1}$, es verdad que rutinas, estilos de trabajo, prácticas estandarizadas se imponen y cristalizan en estas disciplinas al igual que en las ciencias físico-naturales. De tal modo, tendría razón Kuhn sobre Popper: los científicos no son aventureros del pensamiento que arriesgan permanentemente sus certidumbres ante pruebas audaces para buscar que tales certidumbres caigan (y así avanzar hacia hipótesis superadoras) sino que son eminentemente conservadores; prefieren jugar dentro de reglas preestablecidas y mantenerse dentro de las mismas. Les es - "nos” es- difícil cambiar las modalidades de trabajo con las cuales ellos se han familiarizado, y a las cuales les deben aquello que hayan obtenido como reconocimiento.

El corolario que esto implica, es que hay una distancia considerable entre una laxa conciencia respecto de la necesidad de modificar los meca- 
nismos intelectuales e institucionales de funcionamiento de la ciencia, y la decisión y voluntad necesarios para que alguna modificación sistemática se establezca. De tal manera, posiciones como las de Wallerstein o las de los autores poscoloniales -muy diferentes mutuamente, por cierto, aunque articuladas entre sí por algunos autores latinoamericanos- son reconocidas por científicos sociales diversos, pero ello está lejos de significar que hayan servido a cambiar siquiera un poco las prácticas efectivas que se realizan en las instituciones científicas.

De cualquier modo "el malestar en la cultura científica" existe. Es notorio que hay temas de urgencia para los cuales se tiene respuesta débil: la inseguridad ciudadana y el narcotráfico son dos de ellos, mutuamente articulados aunque no coextensivos entre sí. La reconfiguración de los sentidos comunes por los medios masivos es otro tema muy referido, pero no suficientemente esclarecido a pesar de su inmensa importancia para la política y la cultura de la hora. La reconstitución económico-financiera del sistema/mundo en este momento de crisis capitalista y recesión generalizada no aparece suficientemente esclarecida; las nuevas modalidades de especificación de lo ideológico en una sociedad donde la autonomía parcial de lo simbólico se ha reforzado, tampoco están estudiadas en espacios académicos que hace rato olvidaron la categoría de "ideología”.

No faltan temas que se puede apreciar como importantes socialmente, para los cuales no hay suficiente desarrollo relevante de parte de la ciencia social contemporánea. Incluso no la hay de parte del conjunto de saberes científicos de la hora, en la medida en que la ecología ha puesto en cuestión la noción tradicional de ciencia que pensaba la naturaleza como infinitamente explotable, noción que queda en situación de parcial suspensión sin que esté del todo claro cuáles son las opciones alternativas. Los problemas que se dan en el dilema explotación petrolera/ambiente limpio para países pobres (es el caso del Ecuador actualmente), o entre explotación minera y recursos no renovables, dejan claro que la visión anterior es hoy insuficiente, pero que no se ha constituido otra suficientemente detallada y aceptada.

Puede notarse esto en la cuidadosa manera en que Wallerstein disecciona la ciencia contemporánea ${ }^{2}$. El ya había mostrado, a través del Informe Gulbenkian que coordinó, que las ciencias son fruto del siglo XIX, principalmente de la construcción del Estado moderno. De tal modo, están ligadas a su consolidación y mantenimiento, su legitimación y permanencia.

Ciertamente que ese Estado es el que de alguna manera los frankfurtianos y Foucault han mostrado como productor de burocratización de la existencia y disciplinamiento de los cuerpos. La racionalización weberiana del mundo conlleva su elocuente imagen de "la jaula de hierro". Los Estados modernos han producido una gama importante de mecanismos de control social, de procedimientos de archivo y de mirada hacia las prácticas de la población; con ello, la ciencia ha sido parte de un proceso de 
reordenamiento de las actividades, e incluso de los deseos y las subjetividades durante el siglo XX.

Además se ha servido, reclama Wallerstein, a la lógica implacable de la preeminencia del Estado-Nación. Desde ese punto de vista, el autor advierte que se ha tomado como unidad de análisis a las poblaciones ceñidas por un Estado en común, aun cuando desde el punto de vista cultural, territorial y hasta político pudiera haberse tomado unidades de análisis diversas. El Estado-nación se ha impuesto como una unidad naturalizada a quienes analizan lo social, imposibilitando otro tipo de enfoques, sobre todo aquellos que se centran en el sistema socio-económico planetario como un todo.

La idea de Estado nacional ha sido, entonces, propiamente un obstáculo epistemológico, sostiene Wallerstein, para comprender lo que ocurre en el mundo. Ha impedido pensar la totalidad del sistema social y la relación constituyente que existe entre las economías y sociedades de los países centrales y la de los periféricos. Pensando lo limitado a cada Nación no puede entenderse el entramado del sistema y del poder a nivel mundial; a la vez, ello confunde acerca del comportamiento de lo nacional como tal (que es lo que se pone bajo análisis).

Sucede que con la versión de que los estados nacionales resultan prioritarios, tiende a suponerse que la comparación entre los mismos goza de algún estatuto de validez. Consecuentemente se da la división entre "países avanzados” y "atrasados” y la comparación de ambos sin el contexto de su relacionamiento mutuo, lo cual lleva a la ilusión de que algunos son exitosos porque han hecho mejor las cosas, y de que el destino de los que están "atrasados" sería ir pasando por las mismas etapas por las que fueron antes los países avanzados.

No puede dejar de advertirse la enorme pertinencia de esta observación. Efectivamente, a nivel del sentido común socialmente sedimentado, es muy habitual la noción de que los países del capitalismo periférico no llegamos a las posibilidades económico-sociales del capitalismo central por alguna falla histórica en nuestro comportamiento. No seríamos tan éticos, tan efectivos, tan voluntariosos o tan inteligentes como los actores sociales del capitalismo del Norte, los cuales sí asumen la disciplina social e intelectual que resultarían indispensables para “despegar” y progresar, para salir de la pobreza y conseguir el ansiado desarrollo.

La pregnancia ideológica de esta idea es enorme, y los argumentos de sentido común invaden, por ej., al periodismo, en el cual es habitual hallar comentarios instalados en esta dualidad entre los adelantados y los atrasados, entre los aptos y los fracasados, en última instancia entre los blancos y los que pertenecen a las restantes etnias del globo terráqueo.

Los males de quedarse en el análisis exclusivamente nacional para nada terminan allí, denuncia Wallerstein. No sólo quedamos entrampados 
en la idea de imitar al capitalismo avanzado que nos sojuzga y que es mundialmente responsable de la situación de los países oprimidos, sino que incluso concebimos nuestras nociones emancipatorias contra ese sistema dentro de esas mismas ideas que resultan limitativas del conocimiento, así como de la eficacia práctico-política.

En este punto el análisis del referido autor marxista se hace sumamente fino, dado que su posición tiene implicancias político-ideológicas fundamentales. Con amplia argumentación busca demostrar que los procesos políticos de liberación nacional son intrínsecamente contradictorios, y que -al margen de la conciencia subjetiva de sus actores- terminan reforzando los mecanismos del poder al cual se oponen.

Cierto es que muchas de esas revoluciones nacionales han tenido reversiones que las han llevado a posiciones parcialmente restauradoras de las condiciones previas de poder. Si bien ya el colonialismo abierto ha desaparecido, el mismo se renueva en el turismo, la diplomacia, la economía internacionalizada, el intercambio comercial y el colonialismo interno. Si pensamos en un caso como el argelino esa situación se hace patente, y creemos que incluso para el ejemplo de la revolución china puede aplicarse similar argumento.

Contra la noción de que la burocratización sería una condición intrínseca de la política, Wallerstein sostiene que es propia sólo de las políticas estatalistas; de modo que la Unión Soviética siguió el camino inevitable que correspondía al extraño concepto de "socialismo en un solo país".

Podría argumentarse que la revolución proletaria mundial tiene que empezar por algún país concreto, que los nacionalismos configuran en gran medida las identidades de los sectores populares, y que por todo ello una revolución internacionalista como la que Marx pensaba resulta intrínsecamente imposible, al menos dentro de las condiciones de visibilidad histórica que se nos dan en la época que nos toca.

Wallerstein acepta este argumento, pero señala que los atajos que podamos tomar en consecuencia resultan espejismos. Vamos por el camino de lo posible, el de las revoluciones en el marco de los estados nacionales. Pero éstos tienen que retomar relaciones comerciales y financieras capitalistas con el mundo exterior, lo que los obliga a la competitividad internacional y -por ello- a forzar la producción interna y una industrialización intensa. Para ello debe promoverse fuerte disciplinamiento, además de incentivos material-económicos diferenciales hacia distintos segmentos de la población. De tal modo se va reasumiendo la renta diferencial de las clases sociales y el Estado nacional fruto de las guerras de liberación se ve obligado a sostener esa situación, pues está en competencia con otros estados a la vez.

En cuanto al tema del poder, este Estado no tiene mayores posibilidades de colaborar a hacer la revolución en otros países; cuando esa fue la 
política de la revolución cubana, estuvo a punto de caer militarmente con la invasión a Bahía de Cochinos y se agudizó enormemente la conflictividad internacional. Toda revolución nacional ha de producir una defensa de su Nación ante las otras, más que una defensa del socialismo en las mismas; por ello tenderá a establecer modalidades de acumulación lo más competitivas posibles, y ello conlleva lentamente el retorno a relaciones de dominación interna.

Nuestro autor es consciente de que no había otras posibilidades para los movimientos de liberación del siglo XX; no plantea una imaginaria situación por la cual liderazgos antiestatalistas hubieran podido hegemonizar los movimientos populares. Pero señala que era inevitable que ocurriera, entonces, lo que ocurrió: estos movimientos eran lo posible a corto plazo, pero a largo plazo estaban condenados a restaurar las condiciones previas a su existencia.

Desde esta matriz teórico-política es que Wallerstein saca conclusiones epistemológicas, entendiendo que si las ciencias sociales han surgido del auge de los estados nacionales, están ellas mismas inficionadas por errores implícitos de concepción que se hacen inerradicables y estructurales.

Cabría, sin embargo, discutir esa tesis tan sólidamente planteada por el autor. Porque así como en los epistemológico veremos que sus posiciones son más claras a la hora de criticar que a la de proponer, lo mismo podría decirse de su análisis sociopolítico. ¿Qué puede ofrecerse frente a la idea de que la lucha por el poder del estado es equívoca, pero que -asumimos nosotros- dejar el estado en manos de los pro-capitalistas es suicida? Simplemente dejar que el capitalismo lleve las riendas para "profundizar las contradicciones", parece notoriamente absurdo y contraproducente. La idea de algo así como un derrumbe "natural” del capitalismo que cupiera esperar si se lo deja desarrollarse sin resistencia, lleva a la oscura consigna de “cuanto peor, mejor”. Pero nada garantiza que en esas condiciones de fortalecimiento del poder capitalista, las cosas vayan hacia una radicalización de la conciencia en dirección de un necesario cambio. De tal manera, entendemos que la lucha por el poder del estado resulta siempre necesaria.

Quizá Wallerstein no rechazaría esa conclusión; diría solamente que estamos ante un espejismo, ante la fatalidad de que nos metemos en un camino seductor al comienzo pero que fatalmente nos llevará luego al fracaso histórico de las experiencias que se hagan bajo esta decisión de apoderarse del estado dentro del capitalismo, y por ello a reproducir a mediano plazo sus condiciones iniciales.

Lo cierto es que esos estados que no se pliegan totalmente al capitalismo dominante (los de las "revoluciones nacionales" y movimientos de liberación del capitalismo periférico) disminuyen las condiciones de ejercicio del poder económico dentro del capitalismo hegemónico. Es este un punto que no desarrolla nuestro autor; de modo que pareciera, para él, que 
los movimientos de liberación fueran totalmente funcionales al capitalismo central, lo cual no es obvio que se dé de tal manera, en la medida en que es sabido que ese capitalismo central los rechaza fuertemente.

A su vez, la idea de que pueda pasarse a nivel mundial desde el Estado capitalista al no-Estado por rebelión popular masiva y espontánea lo cual parece ser el corolario de las afirmaciones de Wallerstein- es altamente problemática. No sólo es contrafáctica (es decir, no ha ocurrido y si alguna vez llegara a ocurrir no podemos preverlo), sino que poco podemos hacer por ayudar a que ocurra, pues sus condiciones son tan lejanas a las de la política (las cuales todavía se resuelven en ámbitos internos a los Estados-nación) que debiéramos esperar a alguna explosión cuasi-natural de alcance planetario, sin mínima seguridad de que en algún momento ella llegara a darse.

Las aporías que nuestro autor establece sobre los movimientos de liberación son efectivamente existentes, pero de ninguna manera autorizan "por la positiva" a tomar su contrario, despreciar el poder del Estado y dejarlo en manos del capitalismo hegemónico. En todo caso, sí invitan a pensar alguna forma de superación de los problemas que surgen de la toma del poder del Estado en un solo país; los esfuerzos latinoamericanos actuales por lograr integración geopolítica y económica entre países diferentes (ejemplarmente objetivados en la conformación y funcionamiento de CELAC y UNASUR) van en esa dirección, y podrían servir a dos grandes posibilidades a la vez: 1.Control y apoyo mutuo entre los procesos de los diferentes países, de modo de no dejar a los movimientos nacionales sumidos a su sola suerte; 2.Fortalecimiento de las posibilidades económicas de resistir a la presión del capitalismo internacionalizado, por vía del apoyo y coordinación entre diferentes estados.

Wallerstein podría afirmar que eso sería como querer curar el cáncer con aspirinas, y que él plantea una tendencia estructural de los estadosnación que es intrínsecamente problemática.

Y si bien estamos de acuerdo con él al respecto, este autor parece no asumir que ésa es la única direccionalidad histórico-política pensable; lo político no puede "saltarse etapas" por fuera de las condiciones históricas inmanentes, y pasar así -sin mediación- de la lucha contra el estado burgués (mayormente no cumplimentada) a la eliminación planetario-universal del estado como institución. Parece más razonable pensar en términos de dialéctica, por la cual al estado burgués corresponda oponer un estado diferente, desde el cual -ya posteriormente- pueda avanzarse hacia su posterior desaparición.

Podría argumentarse que eso ya se ha realizado, y el estado no tendió a su extinción sino a su reforzamiento. Y que todo estado tendería más a su perpetuación que a su autosupresión. Todo lo cual es cierto; pero no tiene en cuenta la posibilidad de torcer el rumbo que ha habido hasta ahora si el capitalismo a nivel mundial se debilita (actual crisis económica en 
Europa y Estados Unidos) y, sobre todo, si aparece una situación inédita como es la unión tendencial entre diversos estados de raigambre popular en contra de la hegemonía capitalista central.

En fin: Wallerstein podría sostener que la crisis de representación política y su desprestigio avalan la posibilidad de un mundo donde la política-estado desaparezca; pero también podría pensarse que tal crisis de la política tradicional puede significar la abolición de lo político “in toto”, incluyendo cualquier opción anti-sistema (y con ello la perpetuación del Estado capitalista). También puede argumentarse que una política de la pura espontaneidad popular a nivel planetario sin organización y coordinación sistemáticas, podría ir necesariamente a la derrota frente a las fuerzas organizadas del imperialismo.

La discusión político-ideológica queda abierta, y lo menos que pueda decirse es que la aportación de nuestro autor a la misma no sea útil y pertinente. Sin embargo, su capacidad para advertir las limitaciones de lo existente no tiene como correlato la posibilidad de abrir con claridad una opción superadora. No por limitaciones del autor, quizá, sino más bien por condiciones estructurales en el ámbito de lo que aparece actualmente posible a partir de su análisis; lo cierto es que poco queda para hacer tras sus advertencias, si se sigue su postura, salvo esperar una especie de caída espontánea del capitalismo global que nos recuerda a la -nada exitosa por cierto“teoría del derrumbe” vigente hasta hace algunas décadas (que se adscribía a la idea de baja gradual de la tasa de ganancia, según la obra de Marx).

Por todo lo antedicho entendemos que la crítica de Wallerstein a las perspectivas basadas en el Estado-nación, no queda suficientemente sustentada ${ }^{3}$. Si ponerse en el lugar del análisis del sistema/mundo es propender a una transformación planetaria del sistema político que ocurriría de manera espontánea y masiva, cataclísmica e imprevisible, en verdad que es una perspectiva desde la cual las esperanzas que se ponga pueden muy probablemente perderse definitivamente en el vacío y la imposibilidad.

De tal manera, la base teórico-política desde la cual se estructura el punto de vista epistémico de Wallerstein resulta problemática en sí misma. No porque pudiera predicarse de ella que fuera falsa (de ninguna manera), pero sí porque resulta controvertible, sobre todo en su fase propositiva. Frente a la grave problemática de la emancipación frustrada por vía de la toma del Estado en los estados-nación, no aparece alguna concepción menos problemática cuando se prescinde de tal toma del poder del Estado.

La apreciación del Estado como intrínsecamente antiemancipador, resulta además altamente discutible cuando en los países latinoamericanos es hoy el Estado el principal actor que se opone al mercado para mejorar las condiciones socioeconómicas de los sectores sociales subalternos, y cuando la prédica explícita de los líderes de los movimientos populares en la región apela al Estado como principal herramienta de lucha contra lo establecido desde la dominación imperialista ${ }^{4}$. 
Si vamos a las propuestas propiamente epistemológicas de Wallerstein, hallamos un tipo de déficit similar. Su muestra de las limitaciones de la ciencia contemporánea es sumamente sólida: falta de relación entre lo histórico y lo territorial, visión sólo localista de los grandes procesos histórico-sociales, desconocimiento en las ciencias sociales de lo que es propio de las físico-naturales y viceversa, ruptura de la tradición científica en relación con la humanística, aislamiento entre las disciplinas a la hora de la comprensión de la realidad, incomprensión de nuevos procesos sociales en curso que son ajenos a los que existía cuando las ciencias fueron definidas, problemas ambientales que entran en contradicción con la lógica de conjunto (extractivo-productivista) desde la cual se erigieron las ciencias modernas.

Un repertorio de críticas que este autor comenzó a desplegar desde su intervención en la Comisión Gulbenkian. A partir de su participación como director de la misma en los años noventa, este autor de teoría social se asumió gradualmente como una especie de epistemólogo. Es decir, se dedicó a discutir sistemáticamente la cuestión de la base epistemológica de los estudios sociales (y no sólo de ellos), de modo que abrió una nueva veta de actividad intelectual para su obra. Libros completos -por ej. (In)Pensar las Ciencias Sociales- surgieron bajo esta preocupación.

Si bien la desproporción entre un excelente diagnóstico y una débil propuesta se advierte tanto en el Wallerstein científico-social como en el epistemológico, entiendo que no puede pensarse a ambos como ocupando el mismo nivel de definición y calidad conceptual. El científico es el primero, el organizador del marco desde el cual derivadamente apareció el segundo; de modo que tenemos un científico-social que se ha extendido y ha extrapolado sus tesis hacia lo epistemológico, sin haber pasado por una larga tradición de formación en este último espacio de discusiones.

Me permito subrayar ese punto, porque el "Wallerstein epistemólogo" presenta algunas lagunas importantes, que vale la pena señalar. Y si bien las más evidentes aparecen en su menguado repertorio de alternativas a lo hoy existente, también en sus análisis de la ciencia efectivamente dada hay problemas que no podríamos dejar de señalar y enfatizar, dado que no los hemos visto advertidos en ninguna bibliografía.

Tomemos un caso paradigmático, el de la cuestión de que deben reivindicarse los análisis que superen la fragmentación disciplinar. En su afán de celebrar los estudios realizados por fuera de la división entre ciencias, el autor festeja a la vez lo que pueda hacerse en el plano del marxismo y lo que se realiza por vía de los denominados cultural studies(estudios culturales).

Hemos dedicado investigaciones previas a estos estudios acerca de lo cultural $^{5}$, que en su pretendido afán de ser interdisciplinares son a menudo sub-disciplinares (es decir, que no parten de análisis rigurosos practicados desde las disciplinas sino de una conceptualización insípida e incon- 
sistente que pretende prescindir de las mismas, o que apela prioritariamente a una sola por sobre las otras, negando a éstas de hecho). Los estudios culturales pudieron ser promisorios cuando surgieron en el plano del marxismo inglés pero luego fueron derivando hacia la despolitización generalizada, el intra-academicismo asociado a una curiosa retórica de medios masivos, la banalización teórica y la celebración del mercado y el consumo.

¿Cómo asumir a los estudios culturales como ejemplo a seguir en una recomposición de las ciencias sociales? ¿Avala Wallerstein la pérdida de los horizontes ideológicos emancipadores, la trivialización teórica, la mezcla indiscriminada de discursos como ejemplo de práctica interdisciplinar?

El autor ni se ha dado cuenta del problema. Confusamente adscribe a los estudios culturales la misma condición interdisciplinar que él advierte en el marxismo, sin dejar esclarecido que los puntos de vista fundadores de ambas posiciones son epistémicamente incompatibles.

Para el marxismo en realidad no hay interdisciplina, hay segmentación disciplinaria en ciencias sociales que no se condice con la condición del objeto de estudio. La realidad social es una totalidad inescindible, y los aspectos económicos, sociales y políticos se encuentran mutuamente engarzados, de modo que no es posible entender a los unos sin los otros. De tal manera, en las huellas de un pensamiento como el de Lukács ${ }^{6}$ debe pensarse la totalidad como espacios diversos que forman parte de una organización de conjunto, donde la separación entre lo social, lo político y lo económico -como si fueran objetos de disciplinas diferentes- opera como productora de un efecto ideológico de incomprensión de los factores que efectivamente están condicionando lo político desde lo económico, lo económico desde lo político, lo social desde ambos.

Quizá Wallerstein asuma una idea parecida (sólo que "la totalidad” para él es siempre el sistema/mundo, nunca un Estado/Nación). Pero la aceptación de los cultural studies por la débil razón de que ellos se presentan "interdisciplinarios" es muestra de una de las vaguedades epistemológicas propias de las últimas décadas, de las que lamentablemente en el análisis de Wallerstein no hay advertencia. Porque si de algo han de librarse las ciencias sociales contemporáneas, es de esa fácil asunción del espíritu de época posmoderno, de su alivianamiento y su falta de tensión crítica y de exigencia epistemológica. Wallerstein no cede a esa tendencia en sus desarrollos propios, pero sí lo hace al otorgar ese lugar a los "estudios culturales" y al celebrar cerradamente toda presentación de lo que se envase bajo el elástico rótulo de interdisciplina ${ }^{7}$.

Concluyendo: buscar salida a los problemas de la emancipación (la cuestión del Wallerstein “científico”), y sostener una posición epistémica que en su apertura no remita a la debilidad y la falta de rigor, son sin duda opciones a las cuales las ciencias sociales no puedan dejar de remitirse en el horizonte histórico abierto por el siglo XXI. 


\section{Notas}

${ }^{1}$ La obra de Kuhn no remite en casi nada a las ciencias sociales. Ver Kuhn, T.: La estructura de las revoluciones científicas, F.C.E., México, 1980; y nuestro artículo "Sobre la inexistencia de paradigmas en las ciencias sociales" en Follari, R.: Epistemología y sociedad, Homo Sapiens, Rosario, 2000.-

${ }^{2}$ Wallerstein, I.: Saber el mundo, conocer el mundo (el fin de lo aprendido), Siglo XXI, México, 2002

${ }^{3}$ No sólo en este artículo de su libro, pero principalmente en éste desarrolla el autor la cuestión: "El CNA y Sudáfrica (pasado y futuro de los movimientos de liberación en el sistema mundial)”, en Wallerstein, I., ibid., p. 24 y ss.

${ }^{4}$ Ver por ej. las declaraciones de presidentes diversos de la región realizadas en el año 2008 en Filmus, D.: Presidentes (voces de América Latina), Aguilar, Montevideo, 2011

${ }^{5}$ Ver nuestro libro Teorías débiles: para una crítica de la deconstrucción y de los estudios culturales, Homo Sapiens, Rosario, 2002

${ }^{6}$ Lukács, G.: Historia y conciencia de clase, Grijalbo, México, 1969

${ }^{7}$ No pretendemos que no pueda trabajarse en términos interdisciplinarios, ello es no sólo posible sino deseable en muchos y diversos casos; sí advertimos acerca del facilismo con que puede asumirse la práctica de investigación interdisciplinar, la cual exige protocolos y procedimientos epistemológicamente fundados. Ver por ej, nuestro artículo “Acerca de la interdisciplina: posibilidades y límites”, Rev. Interdisciplina, Centro de Investigaciones Interdisciplinarias en Ciencias y Humanidades, UNAM, México D.F., núm. 1, set.-dic. 2013 